\title{
Salidas pedagógicas como metodología de refuerzo en la Enseñanza Secundaria
}

\author{
Meyit Mohamed-Mimón. Universidad de Granada \\ Miguel Ángel Pérez-Castro. Universidad de Granada \\ Miguel Ángel Montero-Alonso. Universidad de Granada
}

Recepción: 23 de marzo de 2017 | Aceptado: 30 de junio de 2017
Correspondencia: Miguel Ángel Montero | Correo-e: mmontero@ugr.es

(iD 0000-0002-1214-9035

Citar: Mohamed, M., Perez, MA. y Montero, MA. (2017). Salidas pedagógicas como metodología de refuerzo en la Enseñanza Secundaria. ReiDoCrea, 6, 194-210.

Resumen: En este artículo se presenta una actividad educativa diseñada para ser realizada fuera del aula, dentro de las diferentes estrategias didácticas que son desarrolladas en el proceso de enseñanzaaprendizaje, y relacionada con la realidad económica. Las salidas pedagógicas, se presentan como una metodología para enfrentarse ante contextos, ámbitos o campos de estudio en los que previamente no existen suficientes recursos didácticos específicos, donde el estudiante conocerá de primera mano y presencialmente, la realidad de un sector económico clave en el entorno donde vive. Además, permite contar con profesionales especialistas de los lugares visitados que facilitarán el correcto desarrollo del proceso educativo del alumnado y donde sus conocimientos prácticos pueden servir a los docentes en la elaboración de recursos didácticos específicos sobre la temática abordada. Mediante una revisión teórica de este proceso, a través de un enfoque constructivista, se analiza el papel protagonista que se le otorga al alumno en estas actividades, generando una dinámica diferente por la que éste es el actor principal y no un ente pasivo de la enseñanza. En la evaluación, se produce un feedback donde se evalúa al alumnado y, a su vez, el alumnado evalúa el proyecto, facilitándose así la mejora continua de la actividad. Los resultados obtenidos proporcionan un mejor desarrollo de los conocimientos económicos del estudiante, habilidades, hábitos y concepción científica del mundo en el que viven, siendo un recurso que potencia su actividad educativa.

Palabras clave: Enseñanza Secundaria | Experimento Educacional

\section{Field Outings as Reinforcement of Teaching Methodology in Secondary Education}

\begin{abstract}
This article presents an educational activity designed to be performed outside the classroom, as part of the different teaching strategies that are carried out in the process of teaching and learning, and related to the particular economic reality. Field outings are presented as a methodology to overcome the lack of specific teaching resources in certain contexts, areas or fields of study. Students learn first hand and in person the reality of a key economic sector in their own environment. The outings also allow professionals from the places they visit to participate in the correct development of the students' learning process, and whose practical knowledge will help teachers in developing specific teaching resources on the topics addressed. Through a theoretical review of this process, from a constructivist approach, the leading role given to students in these activities is analysed, creating a different dynamic in which the student is an active participant, rather than a passive one. In the evaluation, a feedback is provided to evaluate the students and, in turn, the students evaluate the project, facilitating the continuous improvement of the experience. The results show a better development of students' economic knowledge, skills, habits and scientific conception of the world in which they live, being a resource that enhances their learning process.
\end{abstract}

Key words: Secondary Education | Educational Experiment

\section{Introducción}

Conseguir una enseñanza de calidad es un objetivo que persiguen todos los sistemas educativos en cualquier país. La implantación de las materias de Economía y Economía de la Empresa en el bachillerato en España, puso de manifiesto, según Marco (2011), que cuando el alumnado elige estas materias quiere cimentar sus posteriores estudios universitarios y profesionales, debido a que son materias 
reconocidas, suficientemente atractivas y novedosas para conocer social y económicamente el mundo en el que vivimos. Ahora bien, adelantar ciertos conocimientos a la fase educativa anterior, es decir, a la Enseñanza Secundaria Obligatoria (ESO), ha venido marcado por las pautas que el Ministerio de Educación, Cultura y Deporte (MECD) determina y justifica en los contenidos de economía en esta fase formativa.

La economía permite comprender mejor a la sociedad, dando herramientas para la toma de decisiones y dirigiendo el proceso de enseñanza-aprendizaje hacia un resultado final. González (2004) afirma que "la educación económica contribuye a la estabilidad del sistema financiero y tener una cultura en este campo hace que las decisiones de inversión futuras estén mejor informadas y se eviten determinadas situaciones como el sobreendeudamiento". Según Fernández (2012), el nivel de conocimientos financieros en España está por debajo de la media de los países de la OCDE y es sensiblemente inferior al del mundo anglosajón, demostrando con ello la necesidad de adelantar conocimientos financieros prácticos a jóvenes cuyo último contacto con el entorno educativo formal pudiera ser $4^{\circ}$ de la ESO y la finalización de la obligatoriedad educativa.

La Ley Orgánica 8/2013 para la Mejora de la Calidad Educativa (LOMCE) lo argumenta diciendo que la economía está presente en todos los aspectos de nuestra vida cotidiana, y cualquiera de nosotros debería conocer las reglas fundamentales que explican los acontecimientos económicos y la terminología empleada en los informes económicos y en los medios de comunicación. En la vida cotidiana de los alumnos, entender los comportamientos económicos, la distribución de recursos escasos y la forma en que se organizan la producción, distribución y comercialización de bienes y servicios, les ayudarán a su desarrollo como profesionales presentes o futuros y como personas. Cada vez cobran más valor estos conocimientos económicos si se quiere tener ciudadanos solventes e informados, lo que muestra la importancia social de la economía, pues su conocimiento contribuye a fomentar la mejora en la calidad de vida, el progreso y el bienestar social.

Las salidas pedagógicas o actividades fuera del aula, son un recurso pedagógico que ayuda en el aprendizaje de los estudiantes en todos los ámbitos en que se aplique, si son realizadas bajo unos estándares de calidad aceptables, constituyendo una marca de calidad del pasado y del presente educativo, y tienen que seguir siéndolo en el futuro tal y como lo expresa Medir (2003). Algunos autores como Orion (2001) lo definen como actividades que presentan grandes potencialidades para la consecución de los objetivos de la Educación en Ciencias, en la medida en que ocurren generalmente en lugares atractivos; otros como Allen (2004) ven ventajas que se consiguen en ese entorno de trabajo y revelan una experiencia directa con el fenómeno en estudio, armonizando la curiosidad del alumno con una actitud investigativa; o están los que alaban las capacidades personales desarrolladas con dicha herramienta, que proporcionan al alumnado un desarrollo educativo, social y personal o que promueven el conocimiento, las habilidades y actitudes, en el sentido de una mejor percepción y apreciación de los recursos naturales, sin perder de vista su gestión.

Así pues, estudios como el de Romero Ariza (2010), dan especial énfasis a las actividades fuera del aula como potenciador del aprendizaje mediante experiencias vividas en primera persona por el alumnado. CelyRodríguez, Díaz Cáceres y Ocampo Eljaiek (2008) analizan las salidas pedagógicas para un ámbito concreto, la formación en emprendimiento empresarial, exaltando así las ventajas de una formación basada en la experiencia directa con el objeto de estudio consiguiendo así una visión práctica 
y real de las características a las que el alumnado se enfrentara en el mundo profesional.

En el presente artículo se pretende lograr que, mediante la herramienta de las salidas pedagógicas, el alumno pueda estudiar un determinado ámbito del proceso económico de su entorno, ámbito o contexto en el cual previamente no existen recursos didácticos propuestos. Se crea una metodología didáctica que se pueda seguir por los docentes, exaltando la valoración y opinión del alumnado como modo de evaluar y perfeccionar la salida pedagógica en el futuro.

\section{Justificación}

Conocer "in situ" donde se desarrollan muchas de estas actividades humanas, son las razones fundamentales de las salidas fuera del aula, cuya estrategia pedagógica es intentar generar en los jóvenes un manejo globalizado de los conceptos, a la vez que permiten un acercamiento hacia algunas de las realidades sociales y económicas que afectan al entorno donde viven.

El marco de estudio en este caso es Melilla, ciudad española fronteriza situada en el norte de África, considerada un enclave aislado de Europa, que la hacen tener peculiaridades sociales, culturales, económicas, principalmente comerciales y con un régimen fiscal especial, entre otros aspectos. Son estas particularidades las que hacen que el ámbito fiscal y comercial melillense no sea estudiado por sus alumnos en los niveles obligatorios ya que no tienen cabida en los currículos oficiales, y como consecuencia de ello no existen recursos didácticos que se puedan utilizar para la enseñanza de la materia dentro del contexto antes mencionado. Es ahí donde la salida pedagógica aparece como el mejor elemento para dar respuesta didáctica a la actividad que se quiere realizar y donde el alumno podrá aprender en el lugar donde se realizan los hechos y de una manera eminentemente práctica, todo el proceso aduanero y fiscal que atañe a su ciudad.

Por tanto, se pretende llevar a cabo una metodología adecuada conseguir los objetivos que se describen a continuación:

- Definir, como eje del estudio, las virtudes didácticas de las salidas pedagógicas.

- Establecer las salidas pedagógicas como herramientas para afrontar contextos o ámbitos educativos en los que no existan recursos didácticos previos.

- Presentar un sistema de evaluación de éstas que permita una retroalimentación, con el fin de mejorar las salidas futuras, teniendo en cuenta la opinión del alumnado.

- Crear una vivencia educativa para el alumnado de un sector económico estratégico de su ciudad.

\section{Metodología}

En este estudio se parte de la idea de que la salida pedagógica debe intentar cubrir tres de los aspectos más importantes que definen las peculiaridades económicosociales de la ciudad de Melilla. Sus principales características son:

- Ser Ciudad Autónoma.

- Su principal fuente de financiación pública se realiza a través de un impuesto indirecto local único, Impuesto sobre la Producción, los Servicios y la 
Importación (IPSI), que grava la producción, elaboración e importación de toda clase de bienes muebles corporales, las prestaciones de servicios y las entregas de bienes inmuebles situados en la Ciudad de Melilla, en la forma y condiciones previstas en la Ley 8/1991, de 25 de marzo, en el Real DecretoLey 14/1996, de 8 de noviembre, en la Ley 13/1996, de 30 de diciembre, en la Ley $50 / 1998$, de 30 de diciembre, en esta Ordenanza y en cuantas otras disposiciones dicten el Estado o la Ciudad de Melilla para su desarrollo y aplicación.

- Su consideración comercial de ciudad fronteriza con la consideración de territorio franco, fuera de la Unión Aduanera Europea y con las peculiaridades de un comercio exterior, considerado atípico, que viene justificado por la importante diferencia del nivel de riqueza entre ambos lados de la frontera.

Por tanto, metodológicamente se utiliza la herramienta pedagógica de las salidas del aula que permitirá llegar a los objetivos propuestos según los criterios establecidos por la ley. Tal y como dice Morín $(1999,2001)$ :

La enseñanza debe ser desarrollada desde una perspectiva educativa, en la que los contenidos del programa de estudio sean seleccionados, pensados y explorados para promover el desarrollo de la capacidad, actitudes y valores en los alumnos, que les permitan tratar con la incertidumbre, la globalización y la complejidad de las sociedades actuales.

La salida pedagógica se entiende como un instrumento de ayuda al alumno para conocer su entorno, sin que ello suponga ninguna pérdida de calidad en el aprendizaje, teniendo en todo el proceso del aprendizaje contacto con la realidad que se quiere aprender y dejando que el estudiante soporte un proceso lógico de aprendizaje en el que el docente sólo intervendrá para solventar dudas durante el proceso de captación de la realidad aprendida. Investigaciones como las de Pace y Tesi (2004) han demostrado que los alumnos tienen una actitud favorable hacia las actividades que son desarrolladas fuera del aula y donde el uso de estrategias adecuadas potencian el aprendizaje de los estudiantes en este tipo de ambientes y así también lo expresan Orion y Hofstein (1994).

Otros autores como Orion (1993); Rickinson, Dillon y Teamey (2004); Rebar (2009) consideran una serie de obstáculos para poder llevar a cabo esta herramienta pedagógica como: el elevado número de desafíos logísticos a los que los centros educativos someten hoy a los profesores; las presiones a las que estos están sujetos para cumplir el programa de la asignatura; los costes financieros que las salidas ocasionan; el aumento del número de actividades extraacadémicas en las que participan los alumnos diariamente; la suposición de que las salidas de campo son más adecuadas y eficaces para los alumnos más avanzados; y que no todos los profesores están preparados para llevar a cabo estas actividades. Orion (2001) hace especial hincapié en el hecho de que los trabajos de campo generan desafíos adicionales para los profesores, en muchos de los casos que no están familiarizados, la súper-estimulación de los alumnos, causada por la novedad de estos ambientes, puede dar lugar al caos; o las limitaciones del tiempo disponible, o también destacar las dificultades en la elaboración de materiales didácticos adecuados.

La organización de la salida obedece a la desarrollada por Orion (1993), en la medida que tiene un planteamiento tripartito:

1. Psicológico, marcadamente cognitivista. 
2. Epistemológico ya que es muy racionalista al promover, por ejemplo, la pregunta-problema y la observación del pensamiento.

3. Didáctico donde incorpora indicadores de la investigación como el cuestionamiento y la visión holística del conocimiento que se compaginan, tanto con el desarrollo del aprendizaje contextualizado, como con una visión adecuada de la construcción del conocimiento científico, en general, y del sistema en particular.

Esta propuesta de organización, satisfactoriamente demostrada en la bibliografía, contempla las siguientes etapas:

1. Selección de los contenidos y conceptos.

2. Correlación entre los conceptos y las visitas a realizar mediante una planificación de la ruta.

3. Estrategias de enseñanza y aprendizaje con una correlación exacta entre la teoría a conocer y la visión práctica en el lugar donde se materializa.

4. Evaluación del aprendizaje mediante concreción de la importancia de la salida pedagógica.

De este modo, se deben cumplimentar claramente los contenidos y conceptos que se abordan en la salida pedagógica, que deben permitir a los estudiantes alcanzar los objetivos educativos establecidos, por lo que su selección es una etapa importante de la preparación previa. En esta etapa, el profesor se enfrenta a determinar los contenidos que deben ser explicados antes de la salida pedagógica y resumir ideas al finalizar la actividad.

En cuanto al área de estudio, la preparación de la salida pedagógica exige la elaboración de materiales didácticos, incluidos en la guía del alumno, documentos de apoyo al profesor, donde debe aparecer información útil de la salida pedagógica (día, hora, ruta, paradas, duración, lugar de salida, etc.), propuestas de trabajo claras y espacio libre donde el estudiante pueda registrar las observaciones y realizar anotaciones, escribir sus conclusiones y realizar las preguntas o dudas que hayan despertado en él las actividades propuestas.

La salida pedagógica debe estar coherentemente integrada en el programa de estudio, antes y después de la salida, y deben tener una triple función, tal y como dice Fernandes (2005): la integración de las estrategias de la enseñanza seleccionadas por el profesor; ser un medio privilegiado del aprendizaje y estar asociadas a un proceso de evaluación, cuya actividad puede contribuir a una ciudadanía más intervencionista y crítica. Las salidas, al estar integradas en el programa de estudio, deben incluir la evaluación del aprendizaje y ser llevadas a cabo antes, durante y después de la salida.

Estas actividades permiten acercar el aprendizaje realizado en ambientes formales (aula, laboratorio escolar) al que transcurre en ambientes informales (por ejemplo: parque natural, museo, industria, laboratorio de investigación), por lo que el aprendizaje en las salidas difiere del aprendizaje habitual en el aula, en la medida en que ocurre en contacto directo con los fenómenos, en el que están sujetos a una gran variedad de estímulos y donde la evaluación también debe ser diferente. Por otro lado, Rebar (2009) considera que, dependiendo de la finalidad de la salida pedagógica, son varios los datos que pueden entrar en la evaluación de los alumnos, pudiendo algunos de ellos ser recogidos ya en el aula. 
Además, la figura didáctica del portafolio como recurso educativo que ayuda a los estudiantes y permite visualizar su progreso a través de los registros acumulados y los comentarios, les facilita al mismo tiempo la autoevaluación del mismo y a su vez sirve de motivación al estudiante, de ayuda para mejorar su aprendizaje y para crear un hábito de revisión (Barrios 2000).

La evaluación debe ser diseñada de manera que promueva esa interacción. Este tipo de aprendizaje (cognitivo y procedimental) puede también ser evaluado mediante pruebas escritas. Éstas permiten ayudar a que los alumnos presten atención a los detalles de interés y relevancia, teniendo en cuenta la finalidad de la salida y permite facilitar conexiones con los contenidos tratados en el aula y en clases posteriores. Para Rebar (2009), los proyectos, las presentaciones orales y los informes realizados en grupo se integran bien en la experiencia del aprendizaje vivida en este tipo de ambientes. Según el mismo autor, los documentos escritos, dibujos y entrevistas, cuando así los solicite el profesor, han demostrado su eficacia en la captura del crecimiento conceptual de los alumnos que es el resultado del aprendizaje durante las salidas.

En la siguiente tabla (Tabla 1) se enumeran los criterios que deben ser utilizados en la evaluación de los alumnos, las herramientas que pueden emplearse en el proceso, donde será necesario que estén bien definidos sus procedimientos, su ponderación y que éstos sean conocidos por los estudiantes, para un mejor rendimiento en ambientes informales. Ésta se ha elaborado en un formato que permite conocer el plan y la forma de evaluar la salida pedagógica.

Tabla 1. Evaluación de la salida pedagógica.

\begin{tabular}{|c|c|c|c|}
\hline $\begin{array}{l}\text { Situaciones y } \\
\text { ambientes de } \\
\text { aprendizaje }\end{array}$ & Criterios de evaluación & $\begin{array}{l}\text { Herramientas de } \\
\text { evaluación }\end{array}$ & Participantes \\
\hline $\begin{array}{l}\text { Preparación de } \\
\text { la salida } \\
\text { pedagógica }\end{array}$ & $\begin{array}{l}\text { Actividad propuesta } \\
\text { Interés despertado por el alumno } \\
\text { Dudas surgidas ante la } \\
\text { explicación de la salida } \\
\text { pedagógica }\end{array}$ & & Alumno/profesor \\
\hline $\begin{array}{l}\text { Salida } \\
\text { pedagógica }\end{array}$ & $\begin{array}{l}\text { Intervenciones del alumno con el } \\
\text { entorno } \\
\text { Relación interpersonal, } \\
\text { Calidad de conocimientos } \\
\text { compartidos }\end{array}$ & $\begin{array}{l}\text { Registro de observaciones } \\
\text { Anotaciones } \\
\text { Autoevaluación }\end{array}$ & Alumnos \\
\hline $\begin{array}{l}\text { Después de la } \\
\text { salida } \\
\text { pedagógica }\end{array}$ & $\begin{array}{l}\text { Calidad de la reflexión efectuada } \\
\text { Capacidad de comunicar por } \\
\text { escrito }\end{array}$ & $\begin{array}{l}\text { Presentación oral } \\
\text { Mapa de conceptos } \\
\text { Discusión grupal de las } \\
\text { observaciones realizadas } \\
\text { Reflexión escrita individual }\end{array}$ & Alumno/profesor \\
\hline
\end{tabular}

Fuente: Elaboración propia a partir de otros autores.

Para enseñar al alumno a aprender y a descubrir los contenidos por sí mismo, a través de su colaboración se ha definido una ficha pedagógica (Tabla 2) y un modelo de informe de ficha pedagógica de la salida (Tabla 3). 
Tabla 2. Ficha pedagógica.

\begin{tabular}{|c|c|c|}
\hline Título & \multicolumn{2}{|c|}{ Salida para conocer tres pilares fundamentales de la economía de Melilla } \\
\hline \multicolumn{3}{|l|}{ Centro } \\
\hline \multicolumn{3}{|l|}{ Profesores } \\
\hline Duración & \multicolumn{2}{|c|}{5 sesiones de trabajo } \\
\hline Curso(s) & $4^{\circ}$ de la $\mathrm{E}$ & 15 años-16 años \\
\hline Asignatura & \multicolumn{2}{|c|}{ Economía } \\
\hline Objetivo general & \multicolumn{2}{|c|}{ Conocer las peculiaridades comerciales de la ciudad de Melilla } \\
\hline $\begin{array}{l}\text { Descripción del } \\
\text { proyecto }\end{array}$ & \multicolumn{2}{|c|}{$\begin{array}{l}\text { Salida de campo a las tres siguientes localizaciones en Melilla: la estación } \\
\text { portuaria y aduana marítima, la oficina tributaria recaudatoria del Impuesto sobre } \\
\text { la Producción, los Servicios y la Importación (IPSI) y la frontera aduanera } \\
\text { terrestre con Marruecos (Beni-Enzar). }\end{array}$} \\
\hline $\begin{array}{l}\text { Objetivos } \\
\text { específicos }\end{array}$ & \multicolumn{2}{|c|}{$\begin{array}{l}\text { Conocer la labor realizada en el puerto de Melilla y en las empresas que prestan } \\
\text { el servicio de distribución de productos a la ciudad. } \\
\text { Conocer el proceso de tributación sobre las mercancías importadas a Melilla y } \\
\text { tener una visión práctica de la tramitación burocrática de todo el proceso } \\
\text { aduanero. } \\
\text { Conocer la documentación necesaria para la importación de mercancías y } \\
\text { entender el trabajo de la vigilancia aduanera. } \\
\text { Comparar la situación diferencial de nuestra ciudad con la del resto de la Unión } \\
\text { aduanera europea. }\end{array}$} \\
\hline Contenidos & \multicolumn{2}{|c|}{$\begin{array}{l}\text { Bloque 1. Razonamientos básicos para interpretar problemas económicos } \\
\text { provenientes de las relaciones económicas de su entorno. } \\
\text { Bloque 2: identificación de los diversos sectores de la economía de Melilla y las } \\
\text { obligaciones fiscales de sus empresas, y cuyo estándar de aprendizaje es } \\
\text { identificar las obligaciones fiscales de las empresas comercializadoras de } \\
\text { bienes importados en Melilla, señalando el funcionamiento básico de los } \\
\text { impuestos del comercio en general del IPSI en particular. } \\
\text { Bloque 6. La globalización económica y el mercado común europeo. }\end{array}$} \\
\hline Actividades & \multicolumn{2}{|c|}{$\begin{array}{l}1^{a} \text { Sesión: Explicación de los términos que emplearemos recogidos en la ficha } \\
\text { didáctica de la salida pedagógica. Explicación general de la actividad y de } \\
\text { proceso aduanero, y de la metodología a seguir en las siguientes sesiones as } \\
\text { como la confección del portafolio y el trabajo final de exposición, dejando claro } \\
\text { la evaluación y los criterios que se valorarán. } \\
2^{\circ} \text { Sesión: visita guiada a las instalaciones de la estación portuaria de Melilla. Se } \\
\text { conocerán los organismos e instituciones privadas y públicas que } \\
\text { coordinadamente realizan las labores para facilitar el aprovisionamiento y } \\
\text { desplazamientos de mercancías y viajeros hacia o desde nuestra ciudad. } \\
3^{\circ} \text { Sesión: visita se conocerá la labor del Servicio de Recaudación del IPSI de la } \\
\text { Ciudad Autónoma de Melilla, } \\
4^{\circ} \text { Sesión: visita el paso fronterizo de Beni-Enzar y del barrio chino, para } \\
\text { conocer in situ el llamado comercio atípico y la aduana } \\
5^{\circ} \text { Sesión: los alumnos, ya en el aula, expondrán un trabajo en el que explicaran } \\
\text { su portafolio y los documentos necesarios para realizar una operación de } \\
\text { comercio internacional. }\end{array}$} \\
\hline Temporización & \multicolumn{2}{|c|}{ Durante toda una mañana } \\
\hline $\begin{array}{l}\text { Productos } \\
\text { esperados }\end{array}$ & \multicolumn{2}{|c|}{ Portafolios de los alumnos y presentaciones en PowerPoint } \\
\hline Evaluación & \multicolumn{2}{|c|}{ Encuesta de satisfacción a los alumnos evalúen la salida pedagógica } \\
\hline \multirow{2}{*}{$\begin{array}{l}\text { Recursos } \\
\text { tecnológicos } \\
\text { necesarios }\end{array}$} & Hardware & $\begin{array}{l}\text { Pizarra electrónica, móviles de los alumnos para } \\
\text { realizar fotografías, transporte escolar. }\end{array}$ \\
\hline & Software & Enlace a internet \\
\hline Otros & \multicolumn{2}{|c|}{ Materiales corrientes de un estudiante: papel, bolígrafo } \\
\hline
\end{tabular}

Fuente: Elaboración propia a partir de modelos utilizados por los centros. 
Tabla 3. Modelo de informe de la salida pedagógica.

SALIDA PEDAGÓGICA: Estación Portuaria y Aduana Marítima de Melilla, la Oficina Tributaria recaudatoria del Impuesto sobre la Producción, los Servicios y la Importación (IPSI) y la Frontera Aduanera Terrestre con Marruecos (Beni-Enzar)

\begin{tabular}{|l|l|}
\hline Institución Educativa & Instituto de Enseñanza Secundaria \\
\hline Docente Responsable & Profesor del departamento de Economía \\
\hline Grupo realizar la salida & Grupo 1 de $4^{\circ}$ de la ESO \\
\hline Fecha & Un día lectivo. \\
\hline Lugares de Salida & Puerto, Oficina recaudatoria del IPSI y Frontera Beni-Enzar \\
\hline Objetivos de aprendizaje & Defina los indicadores de evaluación de cada meta \\
\hline Objetivo 1 & $\begin{array}{l}\text { Enseñar ideas económicas básicas para que interpreten las relaciones comerciales de } \\
\text { Melilla con Marruecos y el papel de ser territorio franco en un mundo globalizado }\end{array}$ \\
\hline Objetivo 2 & $\begin{array}{l}\text { Enseñar conocimientos de Economía y empresa, identificando diversos sectores clave } \\
\text { de su economía y las obligaciones fiscales de sus empresas }\end{array}$ \\
\hline
\end{tabular}

\section{Actividades de la salida: relacione todas las actividades que realizará con el fin de alcanzar las} metas de aprendizaje

Durante: En el puerto de Melilla se analizan los procesos comerciales de import-export y la misión de todas las empresas que participan en ellos (autoridad portuaria, navieras, estibadores, seguridad y control. El traslado a la Aduana portuaria permite conocer el proceso de tributación y burocracia sobre las mercancías importadas a Melilla y en la Aduana fronteriza terrestre conocer el comercio de exportación, comercio atípico y el trabajo de la vigilancia aduanera

Después: Exposiciones individuales y en grupo en clase sobre los portafolios realizados por los alumnos

\section{EVALUACIÓN}

Describa las lecciones aprendidas de cada momento: Tratamos tres de bloques de la programación de la asignatura: Ideas económicas básicas, Economía y empresa y Economía internacional y a la globalización económica.

Indique y describa los factores que hicieron posible o no el cumplimiento del objetivo

Objetivo 1:

Ver los procesos in situ y escuchar a las personas participantes, lo convierten en una enseñanza multifactorial, no solo sobre aspectos locales, también sobre el significado de globalización y el lugar geoestratégico en que se encuentra Melilla en este mundo.

Objetivo 2: Escuchar la experiencia de las personas y empresas que intervienen en los procesos y como se les explica la importancia de la tributación en la sociedad actual

EVALUACIÓN ACTIVIDADES

Antes:

Durante (pertinencia del lugar visitado en relación con las metas de aprendizaje): Visitar los sitios donde se realizan tres procesos fundamentales para la economía de la ciudad y de su entorno

Después:

¿Qué impacto tuvo la salida pedagógica en la institución educativa?

\begin{tabular}{|l|l|}
\hline EVIDENCIAS & $\begin{array}{l}\text { Relacionarnos con entidades públicas y privadas para que conozcan la labor } \\
\text { formativa del centro sobre las actividades económicas que estos realizan }\end{array}$ \\
\hline INSTRUMENTOS & Realización de presentaciones y documentos para ser explicados en las aulas \\
\hline FOTOS & Elaboración de un reportaje video-fotográfico resumen de la salida \\
\hline EVALUACIONES & $\begin{array}{l}\text { Conseguir en las evaluaciones que los alumnos demuestren no solo tener } \\
\text { conocimientos sobre aspectos teóricos de la materia }\end{array}$ \\
\hline OTROS & $\begin{array}{l}\text { Los padres entienden el esfuerzo de la institución en enseñar a sus hijos la } \\
\text { realidad económica de Melilla de la mejor manera práctica posible. }\end{array}$ \\
\hline
\end{tabular}

FIRMAS DE ESTUDIANTES QUE ASISTIERON

\section{DOCENTE RESPONSABLE}

Fuente: Elaboración propia a partir de modelos utilizados por los centros. 


\section{Desarrollo de los contenidos}

\section{Presentación}

El proyecto está compuesto por cinco sesiones, tres de ellas son salidas guiadas, una a las dependencias aduaneras del puerto de Melilla, otra a los servicios de importación de la hacienda local de la Ciudad Autónoma y la última, a las fronteras y aduana con Marruecos. Las otras sesiones que son en el aula se dividen en la primera sesión que explica de una manera general el proceso de despacho aduanero y los conceptos que necesitan conocerse para que las sesiones guiadas tengan su máximo aprovechamiento. Y la última sesión del proyecto los alumnos exponen de manera breve los conocimientos aprendidos.

\section{Temporización}

Las salidas pedagógicas se programan iniciado el curso y con suficiente anticipación para agrupar actividades en una jornada docente y reestructurar las clases correspondientes en ese día a fechas nuevamente programadas. Las sesiones y los contenidos de las mismas se desarrollan como se explica a continuación:

- $\quad 1^{a}$ Sesión: Explicación de los términos que se emplean y que están recogidos en la ficha didáctica. Consiste en una sesión de explicación general de la actividad y del proceso aduanero, con videos informativos y resolución de preguntas por parte del profesorado. A continuación, se expone la metodología a seguir en las siguientes sesiones, así como la confección del portafolio y el trabajo final de exposición, dejando claro la evaluación y los criterios que se valoran.

- $\quad 2^{a}$ Sesión: Esta sesión, correspondiente a la primera fase de la salida del aula, es una visita guiada a las instalaciones de la estación portuaria de Melilla. Se conocen los organismos e instituciones privadas y públicas que coordinadamente realizan las labores encomendadas para facilitar el aprovisionamiento y desplazamientos de mercancías y viajeros hacia o desde nuestra ciudad. Principalmente, se analiza el servicio de vigilancia aduanera del puerto de Melilla, donde se explica el proceso de inspección aleatoria de contenedores, así como la comprobación documentaria, dando traslado en última instancia al servicio de recaudación a la importación de la Ciudad Autónoma de Melilla. Para ello, se cuenta con la guía del Técnico de la Agencia Estatal de Administración Tributaria (AEAT), responsable de Inspección Aduanera.

- $3^{a}$ Sesión: Durante la salida del aula, en segundo lugar se visita el Servicio de Recaudación del IPSI de la Ciudad Autónoma de Melilla, y se cuenta con la explicación del Director General del Área, donde se analiza todos los documentos necesarios para la importación de productos a la ciudad así como los distintos requisitos necesarios a la exportación.

- $4^{a}$ Sesión: La salida pedagógica finaliza con la visita a los pasos fronterizos entre España y Marruecos de Beni-Enzar (Frontera Aduanera Terrestre) y del Barrio Chino (principal paso fronterizo de comercio atípico), para conocer in situ el llamado "comercio atípico" y la aduana de la Frontera Internacional, viendo el trabajo realizado en estos pasos fronterizos, tanto para la importación hacia nuestra ciudad, como la exportación directa desde el puerto de Melilla hacia Marruecos. 
- 5a Sesión: En la última sesión del proyecto, y ya en el aula, los alumnos exponen un trabajo en el que explican las ideas recogidas en su portafolio y los documentos necesarios para realizar una operación de comercio internacional. Para evaluar los conocimientos adquiridos, los alumnos comprueban, mediante ejercicios, si ha entendido lo expuesto o no, a la vez que van aumentando su propio conocimiento. El trabajo se realiza en grupo, para así incentivar el trabajo en equipo.

Finalizada la actividad, se realiza una encuesta individual (ver Tabla 4) a los estudiantes para conocer su opinión y que sirve de mejora para los siguientes años. Los datos obtenidos en el presente curso se han analizado y mostrado sus resultados en la presente investigación.

\begin{tabular}{|c|c|c|c|c|c|}
\hline \multicolumn{6}{|c|}{ Tabla 4. Ficha de evaluación. Ficha de evaluación de la salida pedagógica } \\
\hline \multicolumn{6}{|c|}{ ( } \\
\hline \multicolumn{6}{|c|}{$\begin{array}{l}\text { En cada una de las preguntas siguientes, rodee con un círculo el número } \\
\text { que mejor se adecúe a su opinión sobre la importancia del asunto en cuestión. } \\
\text { La escala que aparece encima de los números refleja las diferentes opiniones. }\end{array}$} \\
\hline \multirow[t]{2}{*}{ Pregunta } & \multicolumn{5}{|c|}{ Escala de importancia } \\
\hline & $\begin{array}{l}\text { Muy } \\
\text { mala }\end{array}$ & Mala & Regular & Buena & $\begin{array}{c}\text { Muy } \\
\text { buena }\end{array}$ \\
\hline $\begin{array}{l}\text { Valora si entiendes mejor la ciudad en la que vives después de la } \\
\text { actividad realizada }\end{array}$ & 1 & 2 & 3 & 4 & 5 \\
\hline $\begin{array}{l}\text { Valora si las explicaciones de los responsables de la visita al Puerto } \\
\text { de Melilla te han sido útiles }\end{array}$ & 1 & 2 & 3 & 4 & 5 \\
\hline $\begin{array}{l}\text { Valora si las explicaciones de los responsables de la visita al Servicio } \\
\text { de Importación (CAM) te han sido útiles }\end{array}$ & 1 & 2 & 3 & 4 & 5 \\
\hline $\begin{array}{l}\text { Valora si las explicaciones de los responsables de la visita a la } \\
\text { frontera de Beni Enzar te han sido útiles }\end{array}$ & 1 & 2 & 3 & 4 & 5 \\
\hline $\begin{array}{l}\text { Valora si el material didáctico que se te proporcionó para realizar la } \\
\text { actividad fue de utilidad para el desarrollo de ella }\end{array}$ & 1 & 2 & 3 & 4 & 5 \\
\hline $\begin{array}{l}\text { ¿Consideras que el nivel de los contenidos en la actividad realizada, } \\
\text { es el adecuado para un mejor conocimiento de la economía de la } \\
\text { ciudad? }\end{array}$ & 1 & 2 & 3 & 4 & 5 \\
\hline $\begin{array}{l}\text { ¿Crees que el tiempo de duración de la actividad fue el adecuado } \\
\text { para comprender el objetivo de la salida pedagógica? }\end{array}$ & 1 & 2 & 3 & 4 & 5 \\
\hline \multicolumn{6}{|l|}{$\begin{array}{l}\text { ¿Qué aspectos económicos y sociales te han llamado más la } \\
\text { atención en la salida pedagógica? }\end{array}$} \\
\hline \multicolumn{6}{|l|}{$\begin{array}{l}\text { ¿Qué otra actividad te gustaría integrar en la salida pedagógica para } \\
\text { hacerla más dinámica y mejor canalizada al desarrollo de tu } \\
\text { aprendizaje? }\end{array}$} \\
\hline $\begin{array}{l}\text { ¿Qué cuestiones no te han quedado claras después de realizar la } \\
\text { actividad? }\end{array}$ & & & & & \\
\hline
\end{tabular}

\section{Evaluación al alumno}

Para la evaluación de cada estudiante el profesor valora tanto el portafolio presentado así como la exposición que se hace al final de todas las visitas, donde el alumnado explica brevemente el proceso de las mercancías, y todo lo referente a su tributación. Los criterios de evaluación siguen la rúbrica que se muestra en la Tabla 5: 
Tabla 5. Criterios de evaluación

\begin{tabular}{|c|c|c|c|c|c|}
\hline CRITERIO & NIVEL 1 & NIVEL 2 & NIVEL 3 & NIVEL 4 & NIVEL 5 \\
\hline $\begin{array}{l}\text { Importación } \\
\text { de } \\
\text { mercancías } \\
30 \%\end{array}$ & $\begin{array}{l}\text { No conoce el } \\
\text { proceso de } \\
\text { importación }\end{array}$ & $\begin{array}{l}\text { Conoce el } \\
\text { proceso } \\
\text { físico de las } \\
\text { mercancías }\end{array}$ & $\begin{array}{l}\text { Entiende } \\
\text { tanto el } \\
\text { proceso } \\
\text { físico como } \\
\text { algunos } \\
\text { tramites }\end{array}$ & $\begin{array}{l}\text { Es capaz de } \\
\text { realizar los } \\
\text { trámites de } \\
\text { importación por } \\
\text { si solo }\end{array}$ & $\begin{array}{l}\text { Realiza una } \\
\text { importación } \\
\text { sencilla y conoce } \\
\text { de manera } \\
\text { general las } \\
\text { importaciones } \\
\text { más complejas }\end{array}$ \\
\hline $\begin{array}{l}\text { Documentos } \\
\qquad 20 \%\end{array}$ & $\begin{array}{l}\text { No conoce } \\
\text { ningún } \\
\text { documento }\end{array}$ & $\begin{array}{l}\text { Conoce el } \\
\text { DUA y su } \\
\text { función }\end{array}$ & $\begin{array}{l}\text { Sabe cuáles } \\
\text { son los } \\
\text { documentos } \\
\text { necesarios } \\
\text { para la } \\
\text { importación }\end{array}$ & $\begin{array}{l}\text { Expresa la } \\
\text { diferencia entre } \\
\text { los seguros de } \\
\text { transporte. }\end{array}$ & $\begin{array}{l}\text { Entiende la } \\
\text { complejidad de } \\
\text { las operaciones } \\
\text { de comercio } \\
\text { exterior }\end{array}$ \\
\hline $\begin{array}{l}\text { Aduanas } \\
\qquad 20 \%\end{array}$ & $\begin{array}{l}\text { No sabe que } \\
\text { son las } \\
\text { aduanas }\end{array}$ & $\begin{array}{l}\text { Admite la } \\
\text { existencia de } \\
\text { aduanas } \\
\text { pero no } \\
\text { comprende } \\
\text { su función } \\
\end{array}$ & $\begin{array}{l}\text { Entiende el } \\
\text { objetivo de } \\
\text { los servicios } \\
\text { aduaneros }\end{array}$ & $\begin{array}{l}\text { Comprende las } \\
\text { competencias } \\
\text { aduaneras y el } \\
\text { impacto } \\
\text { económico }\end{array}$ & $\begin{array}{l}\text { Entiende el } \\
\text { proceso de } \\
\text { vigilancia y el } \\
\text { trámite aduanero } \\
\text { general }\end{array}$ \\
\hline $\begin{array}{l}\text { Comparación } \\
\text { de Melilla y el } \\
\text { resto del } \\
\text { mundo } \\
30 \%\end{array}$ & $\begin{array}{l}\text { No conoce el } \\
\text { régimen } \\
\text { aduanero } \\
\text { melillense }\end{array}$ & $\begin{array}{l}\text { Admite } \\
\text { diferencias } \\
\text { de Melilla } \\
\text { con el resto }\end{array}$ & $\begin{array}{l}\text { Conoce el } \\
\text { IPSI y su } \\
\text { función }\end{array}$ & $\begin{array}{l}\text { Entiende las } \\
\text { diferencias entre } \\
\text { IPSI e IVA }\end{array}$ & $\begin{array}{l}\text { Alcanza a } \\
\text { entender todas } \\
\text { las diferencias } \\
\text { aduaneras }\end{array}$ \\
\hline
\end{tabular}

Fuente: Elaboración propia a partir de los criterios de evaluación expuestos.

La evaluación se realiza mediante la observación del docente del cumplimiento de los diferentes niveles descritos en la tabla anterior, evaluando a cada alumno/a en función de haber alcanzado los diferentes niveles, luego se pondera según el peso específico de cada criterio de evaluación y finalmente se interpola la calificación obtenida sobre una puntuación máxima de 10 puntos. Las faltas de asistencia sin justificar se penalizan dependiendo del porcentaje de faltas sobre el número de horas lectivas.

\section{Método}

La investigación se realizó con una metodología descriptivo-interpretativa y ex post facto, utilizando un muestreo no probabilístico por conveniencia. La finalidad era conocer la evaluación que dan un grupo de estudiantes de un centro educativo de la ciudad que estudian $4^{\circ}$ de la E.S.O. y que realizaron la actividad. La muestra tomada fue de 30 estudiantes.

Para valorar las salidas pedagógicas, el instrumento de recogida de información fue un cuestionario, según el modelo de la Tabla 4, a un grupo de estudiantes que las ha realizado. Los cuestionarios fueron depurados, numerados y vaciados en una plantilla del programa SPSS 20.

\section{Resultados}

La herramienta formativa de las salidas pedagógicas resulta un potenciador del aprendizaje y coloca al alumnado en el centro del proceso de enseñanza dando un carácter secundario tanto a los recursos auxiliares como a los docentes. Con esta actividad se logra que el alumnado este en contacto directo con el entorno y realiza el proceso de aprendizaje de una manera eminentemente práctica, siempre con auxilio de los profesionales encargados de cada una de los lugares visitados. 
En cuanto a la evaluación, ésta se puede dividir en dos aspectos, una primera clásica que hace el docente al estudiante, consistente en la evaluación mediante los criterios anteriormente descritos, bajo los estándares de cualquier asignatura con relación económica y según la legislación vigente, y una segunda que se encarga de recoger la opinión del alumnado mediante encuestas de evaluación sobre la actividad realizada, y que se analiza mediante el siguiente informe de evaluación:

\section{Informe de evaluación realizada por los estudiantes}

El grupo estaba formado por 12 chicos (40\%) y 18 chicas $(60 \%)$, todos estudiantes que han realizado la salidas pedagógica. En cuanto a la valoración de los conocimientos adquiridos (Tabla 6 ), un $70 \%$ valoran de forma positiva la actividad realizada indicando que le ha permitido conocer mejor la ciudad en la que reside y por tanto, la actividad realizada.

Tabla 6. Valora si entiendes mejor la ciudad en la que vives después de la actividad realizada.

\begin{tabular}{|lccc|}
\hline Valoración & Frecuencia & Porcentaje & Porcentaje acumulado \\
\hline Mala & 4 & 13,3 & 13,3 \\
Regular & 5 & 16,7 & 30,0 \\
Buena & 10 & 33,3 & 63,3 \\
Muy buena & 11 & 36,7 & 100,0 \\
Total & 30 & 100,0 & \\
\hline
\end{tabular}

Fuente: Elaboración propia.

Seguidamente los estudiantes valoraron las explicaciones recibidas en cada una de las visitas que realizaron, siendo las mejores valoradas la visita al Puerto de Melilla y los Servicios de Importación de la CAM con un $73,33 \%$ del alumnado satisfecho, como se puede ver en los siguientes gráficos.

Gráfico 1. Valoración de las explicaciones de la visita al Puerto de Melilla.

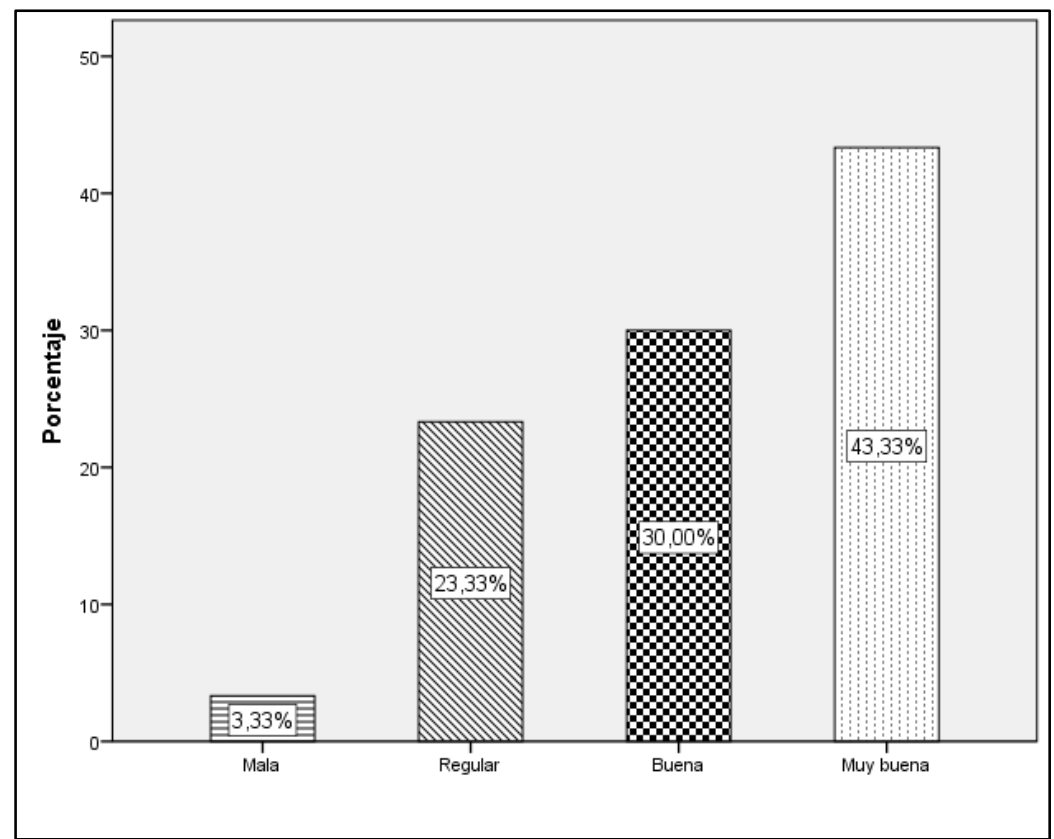

Fuente: Elaboración propia. 
Gráfico 2. Valoración de las explicaciones de la visita al Servicio de Importación (CAM).

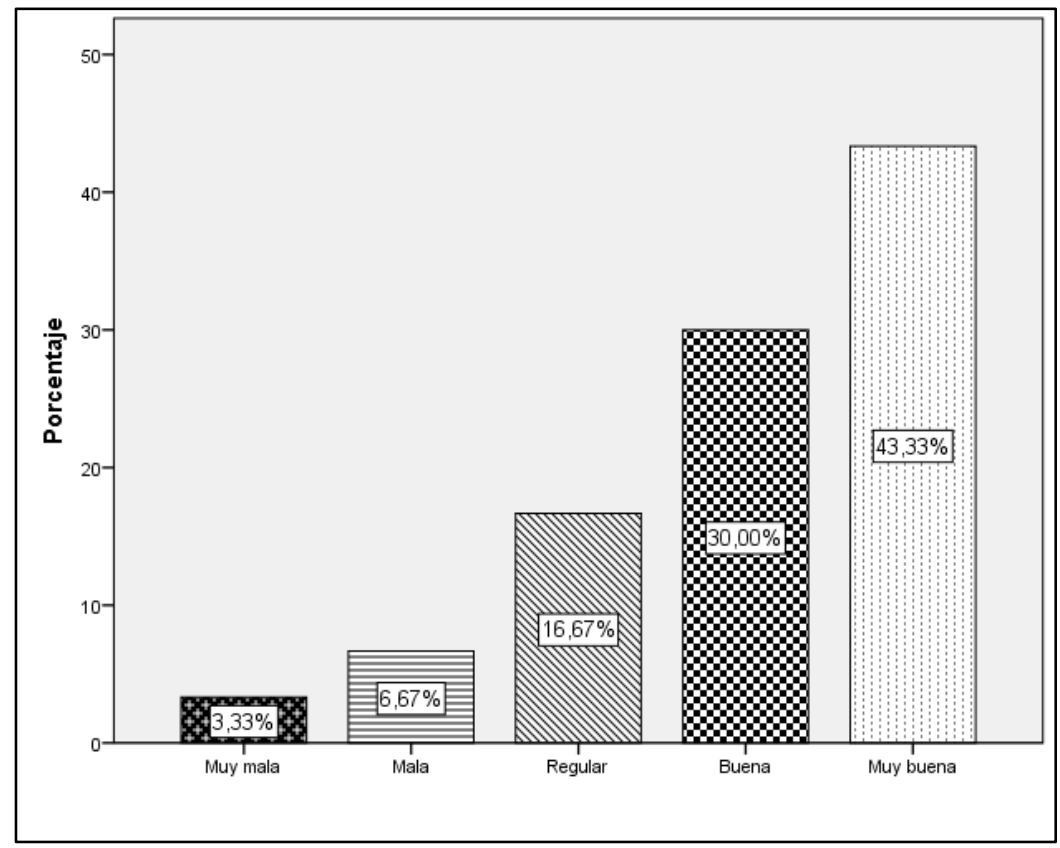

Fuente: Elaboración propia.

Gráfico 3. Valoración de las explicaciones de la visita a la frontera de Beni Enzar.

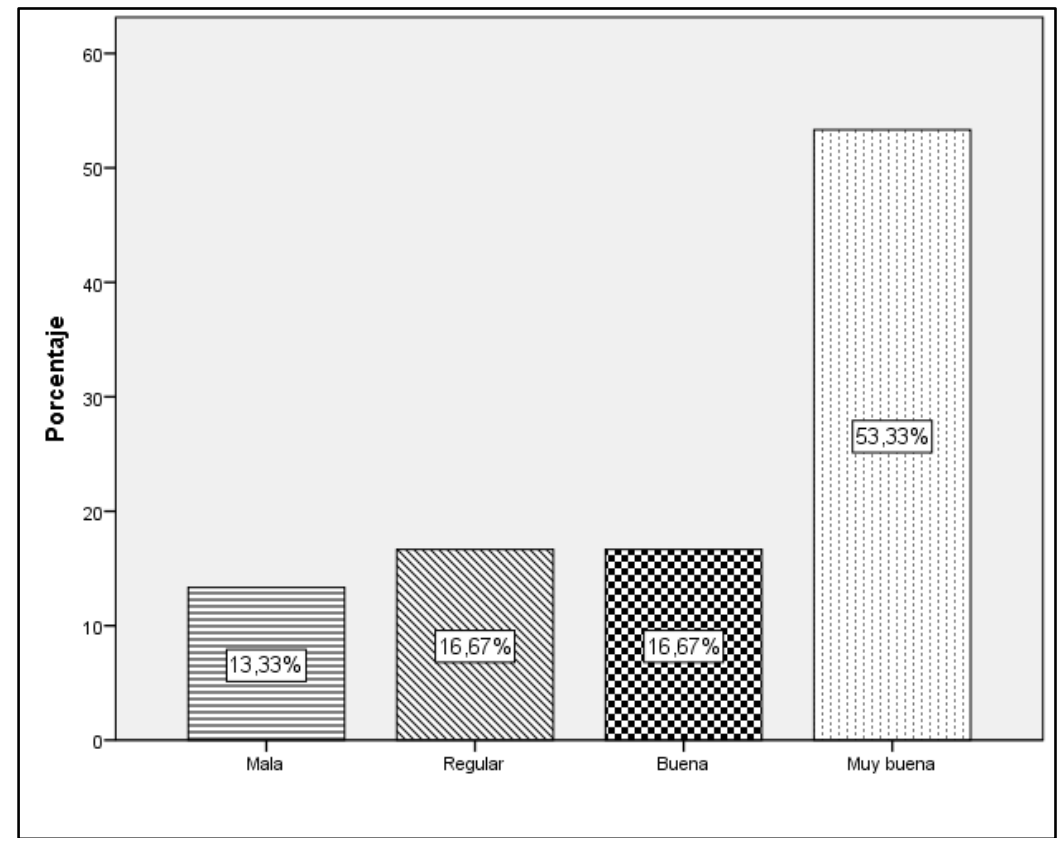

Fuente: Elaboración propia.

Además, como se puede ver en la Tabla 7 , el $80 \%$ del alumnado valora como bueno o muy bueno el material didáctico recibido de los profesionales para realizar la actividad y un porcentaje superior (Gráfico 4) afirma que el nivel de los contenidos es el adecuado para un mejor conocimiento de la economía de la ciudad. Estas opiniones, junto con la evaluación de conocimientos realizados, nos permiten comprobar si realmente han entendido mejor estas actividades realizadas en la ciudad, cuya influencia en la economía de la ciudad es básica en su funcionamiento.

Tabla 7. Valoración del material didáctico que se te proporcionó para realizar la actividad. 


\begin{tabular}{|lccc|}
\hline Valoración & Frecuencia & Porcentaje & Porcentaje acumulado \\
\hline Regular & 6 & 20,0 & 20,0 \\
Buena & 8 & 26,7 & 46,7 \\
Muy buena & 16 & 53,3 & 100,0 \\
Total & 30 & 100,0 & \\
\hline
\end{tabular}

Fuente: Elaboración propia.

Gráfico 4. ¿Consideras que el nivel de los contenidos en la actividad realizada, es el adecuado para un mejor conocimiento de la economía de la ciudad?

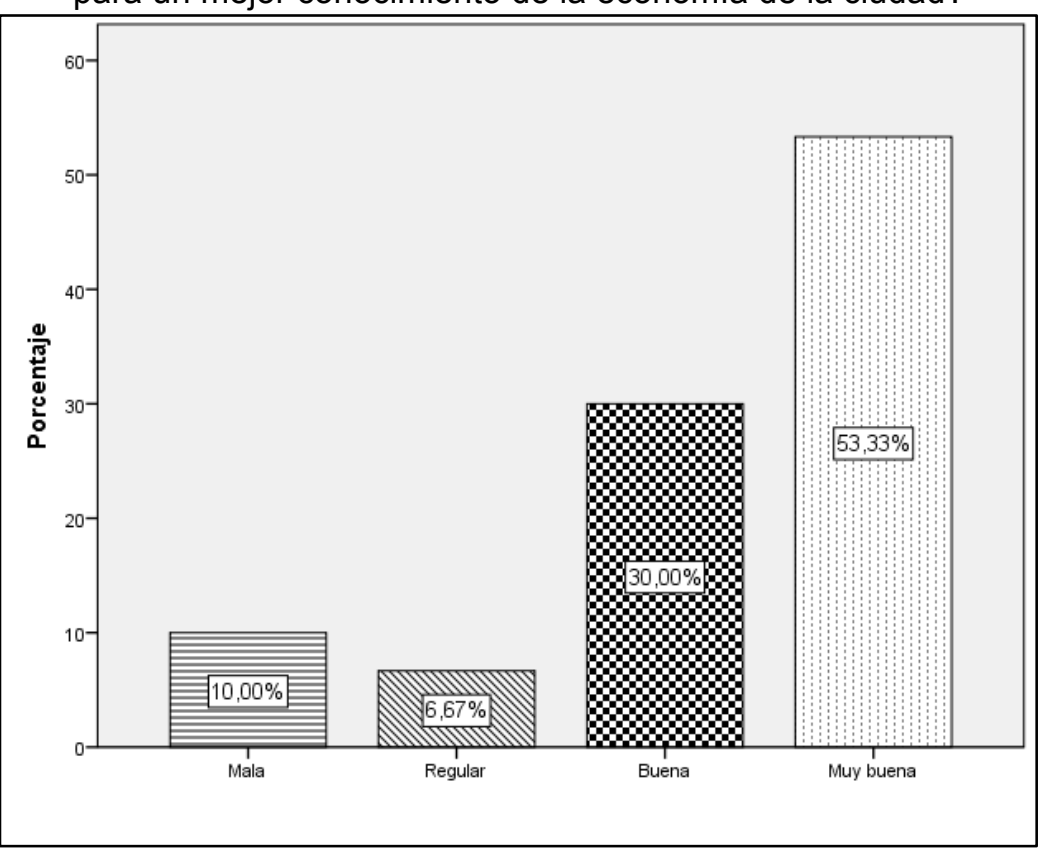

Fuente: Elaboración propia.

Gráfico 5. ¿Crees que el tiempo de duración de la actividad fue el adecuado para comprender el objetivo de la salida pedagógica?

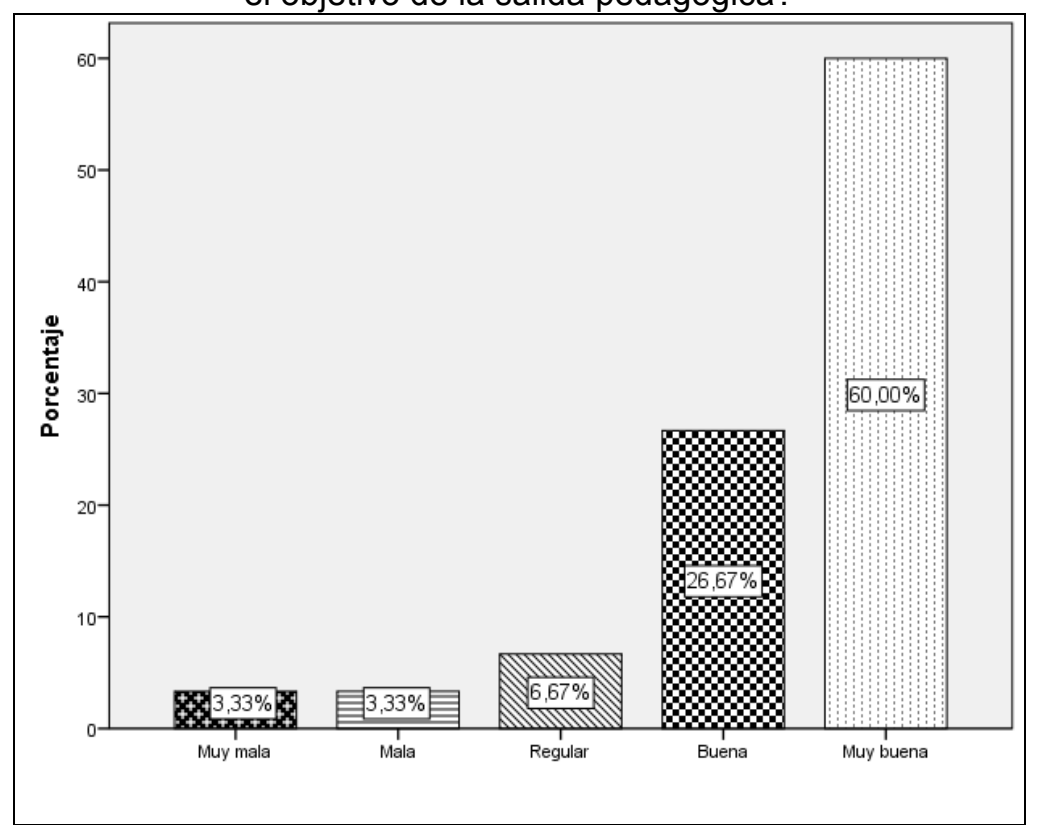

Fuente: Elaboración propia. 
La amplia mayoría, un $86,67 \%$ del alumnado, ha estado satisfecho con la temporalización de la actividad (Gráfico 5).

Gráfico 6. ¿Qué aspectos económicos y sociales te han llamado más la atención en la salida?

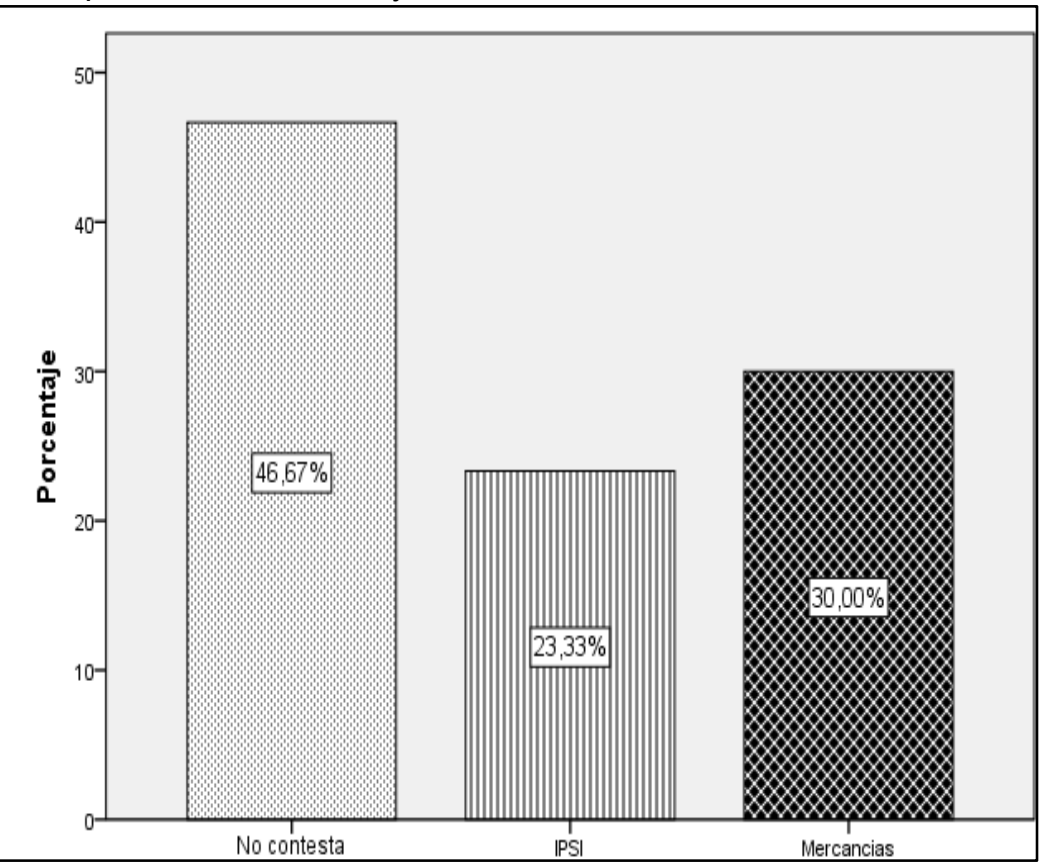

Fuente: Elaboración propia.

En cuanto a los aspectos que más han llamado la atención a los estudiantes, un $30 \%$ destacan el volumen de mercancías y su tratamiento portuario, y un $23,33 \%$ remarcan el IPSI como aspecto a destacar. Ningún estudiante sugiere otra actividad a realizar dentro del marco de esta salida pedagógica y concluyen afirmando que las explicaciones han sido generalmente correctas en cada una de las visitas.

\section{Conclusiones}

Las salidas pedagógicas resultan un elemento potenciador del aprendizaje del alumnado porque se consigue, principalmente, que con la presencia física en una localización concreta y con las explicaciones dadas por los responsables de la actividad, la transmisión practica del conocimiento. Éstas no se realizan con la frecuencia que es recomendada debido a problemas de organización del calendario lectivo o por carecer de fondos para financiar el desplazamiento.

Para el proyecto docente que se presenta, en este caso y dada la situación particular en la que se encuentra, los costes de financiación son prácticamente inexistentes por diversos motivos, entre los que destacan el tener una duración durante el horario lectivo de una jornada y por la cercanía desde el centro de enseñanza a las tres localizaciones que proponemos.

Por tanto, el objetivo de estudio marcado es conseguir que los estudiantes, con una formación y madurez suficiente obtenida al finalizar sus estudios obligatorios de secundaria, entiendan, de una manera general, la importancia económica que tiene para las ciudades autónomas españolas su situación aduanera y comercial.

El comercio es el segundo sector económico por aportación al Producto Interior Bruto (PIB) de estas ciudades, situado después del sector público. Este hecho, junto a que el $100 \%$ de la comercialización de mercancías se realizan a través de importaciones y 
exportaciones, es motivo suficiente para que los alumnos entiendan los trámites necesarios para el proceso de gestión y la documentación aduanera necesaria. El conocimiento de las peculiaridades territoriales de la ciudad y las diferencias existentes con el resto de territorios que si forman parte de la Unión Aduanera Europea, ayudarán a entender muchas de las realidades económicas del lugar donde viven. Este es un contenido que es tratado en parte en la programación didáctica de la asignatura de Economía, y en concreto en tres de sus bloques: Ideas económicas básicas (con razonamientos básicos para interpretar problemas económicos provenientes de las relaciones económicas del entorno de Melilla y el norte de África), Economía y empresa (identificando los diversos sectores de la economía de Melilla y las obligaciones fiscales de sus empresas, señalando el funcionamiento básico de los impuestos del comercio, en general, y del IPSI en particular) y Economía internacional (globalización económica y el papel comercial que realiza el puerto marítimo y la frontera terrestre).

Los estudiantes desarrollarán las capacidades de autoaprendizaje y crítica durante la salida pedagógica y posteriormente, en la doble evaluación que será realizada, el profesorado valorará al estudiante y el estudiante evaluará el proyecto, creándose un proceso de retroalimentación.

Destacar por un lado, que el alumnado ha valorado muy positivamente la salida pedagógica, evidenciándose que la actividad conlleva un gran interés para los estudiantes en cada una de las visitas, agradeciendo también que el aprendizaje se haya realizado fuera del aula. Se demuestra que los alumnos que han participado en la actividad reconocen tener más conocimientos sobre el funcionamiento económico de su ciudad. Por otro, la tarea educativa realizada por los profesionales que atendieron a los alumnos en cada localización, ya que la preparación y posterior explicación de todos los aspectos prácticos para dar a conocer su experiencia profesional ha sido muy enriquecedora para la formación de los alumnos.

\section{Discusión}

En la mayoría de los estudios realizados sobre las salidas pedagógicas como Narváez, García, Guerrero y Modesto (2007); Padierna y González (2013) se ensalza su figura como potenciador del aprendizaje y se realiza una adaptación de una salida a cualquier ámbito del conocimiento. En dichos estudios se potencian los buenos resultados que se consiguen poniendo en práctica la metodología de las salidas pedagógicas, pero a diferencia de ellos, en ninguno de ellos trata de llevar a cabo una evaluación de la salida por parte de quienes son los usuarios finales de toda la actividad educativa, los estudiantes, por lo que se considera de vital importancia ese feedback para la mejora continua de la actividad y, sobre todo, en las actividades que se realizan en espacios no convencionales.

Además, la salida pedagógica se ha potenciado con la figura del portafolio, que de acuerdo con Cabero, López y Jaén (2013), sirve para ayudar a crear la capacidad crítica y autocritica del alumnado y permite comprobar el progreso en el aprendizaje en todas sus etapas, todo ello conseguido en un entorno virtual mediante portafolios digitales. En el trabajo se pretende que mediante su portafolio cada estudiante o grupo de estudiantes tengan la posibilidad de ir recogiendo todas las evidencias posibles de las experiencias vividas en cada visita y que les sirva de recordatorio de toda la salida a la hora de realizar su trabajo final.

Los resultados son muy claros para mostrar que en la muestra analizada, los estudiantes han valorado muy positivamente las salidas pedagógicas propuestas, su 


\section{temporalización y metodología, así como los contenidos y las explicaciones realizadas por los profesionales que les atendieron en cada localización.}

\section{Referencias}

Allen, S. (2004). Designs for Learning: Studying Science Museum Exhibits That Do More Than Entertain. Science Education, 88(1), 17-33

Barrios, O. (2000). Estrategias del portafolio del alumnado. En S. de la Torre y 0 . Barrios (Coords). Estrategias didácticas innovadoras. Recursos para la formación y el cambio. Barcelona: Octaedro.

Cabero Almenara, J., López Meneses, E. y Jaén Martínez, A. (2013). Los portafolios educativos virtuales en las aulas universitarias. Instrumentos didácticos para la innovación docente y la calidad de los procesos de enseñanza y aprendizaje. Enseñanza \& Teaching, 31, 43-70.

CelyRodríguez, A., Díaz Cáceres, N. y Ocampo Eljaiek, D. (2008). Salidas de campo en la formación de emprendedores. Revista Escuela de Administración de Negocios, 64, 101-126.

Fernandes, D. (2005). Avaliação das Aprendizagens: Desafios às Teorias, Práticas e Políticas. Texto Editora. Lisboa.

Fernández, D. (2012). Un país de analfabetos. El país. http://economia.elpais.com/economia/2012/05/04/actualidad/1 336131931 321579.html

González, F. (2004). Las materias de economía en la enseñanza secundaria: una oportunidad para el desarrollo de las capacidades emprendedoras de nuestros jóvenes. http://www.eumed.net/ce/2004/fjga-secund.htm

Ley de Educación. Ley Orgánica 2/2006, de 3 de mayo.

Ley Orgánica 8/2013, de 9 de diciembre, para la mejora de la calidad educativa.

Marco, M. y Molina, J. (2010). La enseñanza de Economía en Secundaria obligatoria y Bachillerato: un factor estratégico pendiente de desarrollo. Economistas, 28 (125), 25-34.

Medir, RM. (2003). Salir de la escuela: entre la tradición y la educación ambiental para la sostenibilidad. Íber: Didáctica de las Ciencias Sociales, Geografía e Historia, IX(36), 26-35.

Morin, E. (1999). Repensar a Reforma. Reformar o Pensamento. A Cabeça Bem Feita. Instituto Piaget. Lisboa.
Morin, E. (2001). O Desafio do Século XXI. Religar os Conhecimentos. Instituto Piaget. Lisboa.

Narváez, J., García, G., Guerrero, P. y Modesto, MC. (2007). Salidas pedagógicas y las TIC's: experiencias docentes. http://colegios.redp.edu.co/restrepomillan.

OCDE. (2013). Propuesta para la mejora de la calidad educativa. España: Ministerio de Educación Cultura y Deporte.

Orion, N. (1993). A model for the development and implementation of field trips as an integral part of the science curriculum. School Science \& Mathematics, 93(6), 325-331.

Orion, N. (2001). A educação em Ciências da Terra: da teoria à prática - implementação de novas estratégias de ensino em diferentes ambientes de aprendizagem. In Marques y Praia, J. (Coords.) Geociencias nos currículos ensinos básico e secundário. Universidade de Aveiro, Aveiro, (93-114).

Orion, N., \& Hofstein, A. (1994). Factors that influence learning during a scientific field trip in a natural environment. Journal of Research in Science Teaching, 31(10), 1097-1119.

Pace, S., \& Tesi, R. (2004). Adult's perception of field trips taken within grades K-12: Eight case studies in the New York metropolitan area. Education, 125(1), 30-40.

Padierna Cardona, JC. y González Palacio, EV. (2013). La metodología experiencial en la Educación Superior. Itinerario Educativo. 62, 151-168.

Rebar, B. (2009). Evidence, Explanations, and Recommendations for Teachers' Field Trip Strategies. Doctoral Thesis. Oregon State University: Oregon.

Rickinson, M., Dillon, J., \& Teamey, K., (2004). A Review of Research on Outdoor Learning. Shrewsbury: National Foundation for Educational Research and King's College.

Romero Ariza, M. (2010). El aprendizaje experiencial y las nuevas demandas formativas. Revista de Antropología experimental, 10, 89-101. 